\title{
Multielectron Spectroscopy : Auger decays of the Argon 2p hole
}

\author{
P. Lablanquie, ${ }^{1}$ L. Andric, ${ }^{1}$ J. Palaudoux,${ }^{1}$ U. Becker, ${ }^{2}$ M. Braune, ${ }^{2}$ J. Viefhaus,${ }^{2}$ \\ J. H. D. Eland, ${ }^{3}$ and F. Penent, ${ }^{1}$ \\ ${ }^{1}$ LCP-MR, Université Pierre et Marie Curie-Paris 6 et CNRS (UMR 7614), 11 rue Pierre et \\ Marie Curie, 75231 Paris, France \\ ${ }^{2}$ Fritz-Haber-Institut der Max-Planck-Gesellschaft, Faradayweg 4-6, 14195 Berlin, Germany \\ ${ }^{3}$ Physical \& Theoretical Chemistry Laboratory, South Parks Road, Oxford OXI 3QZ, UK
}

\begin{abstract}
All the different Auger decay paths of Argon 2p holes have been characterized using a time of flight spectrometer of the magnetic bottle type. All electrons (the photoelectron and up to three Auger electrons) are detected in coincidence and resolved in energy. Double Auger decay is shown to proceed either through a direct process or by intense cascade paths, implying highly excited autoionizing $\mathrm{Ar}^{2+}$ states, which are identified as $\mathrm{Ar}^{2+} 3 \mathrm{~s}^{-2}$ correlation satellites. Triple Auger decay is also observed and estimated to account for $0.2 \%$ only of all Auger decay.
\end{abstract}




\section{Introduction}

Absorption of an energetic photon by a target should in first approximation lead to the ejection of one electron only, as this interaction is purely mono-electronic. However it is well established that sometimes several electrons can be ejected. This can happen in valence multiple ionization or after inner shell ionization when secondary electrons are emitted sequentially (cascade Auger decays) or simultaneously (direct double Auger decays). Such processes and especially the 'one-step' or direct ones (direct double ionization or direct double Auger decay) are extremely interesting to investigate as they reveal the strong electron correlation phenomena which are at their origin. Indeed detailed studies have appeared recently since the introduction of electron / electron coincidence techniques in the field ${ }^{1}$. A partial and non exhaustive list of recent electron / electron coincidence experiments includes valence double ionization of atoms ${ }^{2}$ and especially of $\mathrm{He}^{3,4}$, of molecules ${ }^{2,5,6}$ and especially of $\mathrm{H}_{2},{ }^{7,8}$ and coincidence studies of Auger $9,10,11,12,13,14,15$ and double Auger decays ${ }^{16,17}$. However in most of these examples only a small part of all the processes releasing several electrons is explored as most of the studies have restricted their coincidence detection system or the event analysis to processes where exactly 2 electrons are emitted. A significant progress occurred with the introduction by J. Eland et $\mathrm{al}^{18}$ of a new magnetic bottle time of flight spectrometer ${ }^{19}$. Its advantage lies on the fact that all the electrons emitted over the $4 \pi$ solid angle are detected and analyzed in energy with high resolution. It is then possible to measure electron coincidences with high efficiency and to deduce multi-dimension photoelectron spectra, with as many dimensions as ejected electrons. The use of this new spectrometer revealed the different routes to valence double ionization in rare gas atoms ${ }^{15}$ and molecules ${ }^{20}$, allowed the characterization of double Auger decays in Xe atoms ${ }^{21}$ and the study of core-valence double ionization ${ }^{22}$. 
In the present paper we show results obtained with this new spectrometer and with the experimental approach introduced for our study of the double Auger decay of the Xenon $4 \mathrm{~d}$ holes $^{21}$, to investigate the Auger decays of the $2 p$ holes in Argon. Since its first observation in the sixties ${ }^{23}$, the non coincidence $\mathrm{L}_{2,3}-\mathrm{MM}$ Auger spectrum has been well studied both experimentally and theoretically (see the detailed study of Pulkkinen $\mathrm{et}^{\mathrm{al}^{24}}$, the cascade calculations by Kochur et $\mathrm{al}^{25}$ and the references included). However much less is known on the $\mathrm{L}_{2,3}$-MMM double Auger decay of the Argon 2p holes, which was first detected by Carlson and Krause 40 years ago ${ }^{26}$. It was observed by electron / ion coincidences that $2 \mathrm{p}$ holes can decay by emitting two Auger electrons with a $10 \%$ probability $^{27}$, and evidence was found of the existence of a direct double Auger emission ${ }^{17}$. But a detailed analysis of the Auger spectrum with respect to all possible multi-electron processes is still missing. We show here that our coincidence technique enables one to extract such information. It allows the observation of decay processes for different selected ionic intermediate states including excited satellite states as well as triple Auger emission.

\section{Experiment}

The experiment was performed at BESSY on beam line UE56/2, during single bunch operation of the synchrotron, which provides light pulses every $800.5 \mathrm{~ns}$. The time of flight spectrometer is smaller $(2.4 \mathrm{~m}$ instead of $5 \mathrm{~m})$ than in the original design by Eland et al ${ }^{18}$ and was

described elsewhere ${ }^{21,28}$. Briefly, the source volume is located close to a strong $(0.5 \mathrm{~T})$ permanent magnet. All the produced electrons, except those emitted in a small loss cone towards the permanent magnet, are collected and guided through a $2.4 \mathrm{~m}$ long solenoid $(5 \mathrm{G})$ towards the detector. A double-layer $\mu$-metal shield protects the solenoid from the geomagnetic field. The 
detector consists in a set of micro channel plates of $40 \mathrm{~mm}$ active diameter followed by a phosphor screen used to visualize the image of electron impacts. This image is an enlarged view of the interaction zone with a magnification of around 20 , given by the square root of the magnetic field ratios in the source volume and in the solenoid ${ }^{19}$. Centering of the image on the detector is ensured by adjusting the position of the permanent magnet, mounted on a xyz manipulator. A multi-hit TDC with 250ps time resolution collects the arrival time of electrons and references them with respect to the light bunches. Typical count rates were maintained below $5 \mathrm{kHz}$ in order to minimize false coincidences. A small repelling potential is applied on the permanent magnet in order to allow zero energy electrons to reach the detector in a finite time (5.6 $\mu$ s here). The absolute times of flight of individual electrons cannot be measured in these conditions and can only be obtained modulo the $800.5 \mathrm{~ns}$ light pulse interval. Absolute times of flight can however be recovered for all electrons in coincidence events which contain a $2 p$ photoelectron of known kinetic energy and time of flight. The dead time after detection of a first electron was reduced to $18 \mathrm{~ns}$ compared to the previous configuration of $150 \mathrm{~ns}^{21}$ but still prevents the detection of electrons having almost equal kinetic energies. Conversion from the time of flight $t$ to electron kinetic energy $E$ is reasonably well achieved with a simple formula of the type:

$$
t=t_{0}+\frac{D}{\sqrt{E+E_{0}}}
$$

where $D$ is the flight length and $t_{0}$ and $E_{0}$ are adjustable parameters. Calibration was performed by measuring He photoelectron spectra at known photon energies, and showed improvement with the inclusion of two small correcting higher order terms $\frac{A}{\left(E+E_{0}\right)^{3 / 2}}$ and $\frac{B}{\left(E+E_{0}\right)^{5 / 2}}$. The energy broadening of the peaks, $\Delta E$, deduced from measurements in He is displayed in Fig. 1. It is found that the resolution of the apparatus $\Delta E$ / $E$ is roughly constant at $1.6 \%$. The data 
points deviate from that law for electrons of less than $1 \mathrm{eV}$ as $\Delta E$ is limited to some $8 \mathrm{meV}$, probably because of the limited stability and uniformity of the electric potentials. An estimated overall detection efficiency of the apparatus as a function of the electron energy was obtained by measuring Argon 2p spectra at different excess energies, and by comparing the number of Auger electrons measured with and without coincidence with the $2 p$ photoelectron. Detection efficiency was found to be independent of the electron energy up to at least $100 \mathrm{eV}$ and to decrease only slightly for $200 \mathrm{eV}$ electrons, which are the faster ones produced in Ar 2p Auger decays. The obtained value of $49 \pm 5 \%$ obtained for the overall detection efficiency corresponds to the combination of the detection efficiency of the micro channel plates times the transmission (85\%) of the grid placed in front of them. It suggests that the collection efficiency of the spectrometer itself is effectively close to $100 \%$ for electrons of less than $100 \mathrm{eV}$, and corresponds to a loss cone of less than $15^{\circ}$.

\section{Results and discussion}

\subsection{Filtering of Auger spectra according to the $2 p_{3 / 2}$ or $2 p_{1 / 2}$ hole}

A multi-coincidence spectrum has been obtained at a photon energy of $337 \mathrm{eV}$. This excitation energy is sufficient to create $2 p$ holes in Argon which have binding energies of $248.628 \mathrm{eV}\left(2 \mathrm{p}_{3 / 2}\right)$ and $250.776 \mathrm{eV}\left(2 \mathrm{p}_{1 / 2}\right)^{29}$, but also the Ar $2 \mathrm{p}$ shake-up states of binding energies between 270 and $300 \mathrm{eV}^{30,31}$ and the $2 \mathrm{~s}$ hole of $326.25 \mathrm{eV}$ binding energy ${ }^{32}$. Thus the non coincident Auger spectrum presented in Fig. 2 contains contribution of the decay of all these states. At this photon energy, the $2 \mathrm{p}_{3 / 2}$ and $2 \mathrm{p}_{1 / 2}$ photoelectrons have kinetic energies of 88.4 and $86.2 \mathrm{eV}$, respectively and can be separated with our energy resolution of $\Delta \mathrm{E}=1.4 \mathrm{eV}$. Coincidence with the $2 \mathrm{p}$ photoelectrons then enables us to filter out the Auger spectrum, and to 
extract the individual $\mathrm{L}_{3} \mathrm{MM}$ and $\mathrm{L}_{2} \mathrm{MM}$ components associated to the decay of the $2 \mathrm{p}_{3 / 2}$ and $2 \mathrm{p}_{1 / 2}$ holes, as presented in Fig. 2. They are compared to the non coincident $\mathrm{L}_{2,3} \mathrm{MM}$ Auger spectrum of Pulkkinen et $\mathrm{al}^{24}$, which has been obtained with a $265 \mathrm{eV}$ excitation energy, below the binding energies of shake-up states in order to isolate decay of the $2 p$ holes only. Our results are in good agreement taking into account our much worse resolution (here $2.9 \mathrm{eV}$ as estimated from the $\mathrm{L}_{3} \mathrm{M}_{1} \mathrm{M}_{2,3}$ lines). However, they show the power of the coincidence technique to isolate overlapping components of the Auger spectrum. This is especially clear here for the $L_{3} M_{1} M_{1}$ group (enlarged spectrum in Fig 2), see also the work by Ricz et al on the $\mathrm{LM}_{2,3} \mathrm{M}_{2,3}$ lines ${ }^{33}$. The Auger spectrum for the decay of $2 p$ shake up satellites can also be extracted and is reported in Fig.2. It is found to be of comparable intensity with that of the $2 \mathrm{p}_{1 / 2}$ decay at this photon energy, and reveals energetic components at $229 \mathrm{eV}$.

The strength of the present coincidence method is also to reveal the very weak Auger lines at lower kinetic energies (corresponding to higher excited final states) and to extract them efficiently from the background; as shown in the enlarged coincident Auger spectra in Fig 3. The $\mathrm{L}_{3} \mathrm{MM}$ and $\mathrm{L}_{2} \mathrm{MM}$ spectra have been plotted as a function of the binding energy of the final state, and appear to be almost identical at this resolution. The different branching ratios for the $\mathrm{Ar}^{2+}$ $3 \mathrm{p}^{-2}$ final states ${ }^{24}$ are hidden in the unresolved $\mathrm{LM}_{2,3} \mathrm{M}_{2,3}$ lines, but differences are observed for the $\mathrm{Ar}^{2+} 3 \mathrm{~s}^{-1} 3 \mathrm{p}^{-1}$ final states $\left(\mathrm{LM}_{1} \mathrm{M}_{2,3}\right.$ lines). New Auger lines are observed in Fig. 3 in the zone where it is possible to have access to triply charged $\mathrm{Ar}^{3+}$ ions, whose threshold is reported at $84.124 \mathrm{eV}^{34}$. The question is whether double Auger processes contribute here or not. 


\subsection{Double Auger decay}

The double Auger two dimensional maps associated with the decay of the $2 p$ holes are represented in Fig. 4. All the events where a $2 p$ photoelectron and two Auger electrons were detected in coincidence are considered here. Sorting of the double Auger spectrum according to the $2 p_{3 / 2}$ or $2 p_{1 / 2}$ hole is then immediate, contrary to the work by Viefhaus et a ${ }^{17}$ where only two electrons are observed in coincidence at a time. A blind band for energies between 80 and $95 \mathrm{eV}$ corresponds to the overlap with the $2 p$ photoelectrons: Auger electrons of these energies have times of flight similar to the photoelectrons and cannot be separated from them. The two dimensional surfaces of Fig. 4 show lines of constant Auger energy sum corresponding to different $\mathrm{Ar}^{3+}$ final states. These are revealed in Fig. 5 by projecting Fig. 4 along the $\mathrm{x}=\mathrm{y}$ diagonal. The projections can be scaled as a function of the sum of Auger kinetic energies or as a function of the binding energy of the $\mathrm{Ar}^{3+}$ final states, by using values of the $2 \mathrm{p}$ binding energies from the literature $^{29}$. The shapes of both $2 p_{3 / 2}$ and $2 p_{1 / 2}$ double Auger spectra appear to be similar, suggesting similar branching ratios towards the $\mathrm{Ar}^{3+}$ final states. Comparison with reported $\mathrm{Ar}^{3+}$ levels ${ }^{34}$ demonstrate that $3 \mathrm{p}^{-3}$ states are predominantly populated, followed by weaker $3 \mathrm{~s}^{-1} 3 \mathrm{p}^{-2}$ lines and by broad unresolved bands of weak lines corresponding to $\mathrm{Ar}^{3+}$ satellite states of $3 s^{2} 3 p^{2} n l$ configurations, with $\mathrm{nl}=3 \mathrm{~d}, 4 \mathrm{~s}$ or $4 \mathrm{p}$. Similarity to what is observed for the single Auger spectrum in Fig. 3 is striking.

Intensity along the lines in Fig. 4 corresponding to the $\mathrm{Ar}^{3+} 3 \mathrm{p}^{-3}$ final states is strongly structured as revealed by their projection on the $\mathrm{x}$ and $\mathrm{y}$ scale in Fig. 6 . A U-shape is observed with strong indirect processes (cascade Auger decays) contributing at high Auger energies and at the symmetric low energy zone. A weaker unstructured continuum covers the whole Auger energy range and originates from a direct double Auger process where the 2 Auger electrons are 
simultaneously released. The nature of this process was demonstrated by angular resolved coincidences of the Auger electron pairs ${ }^{17}$.

\subsection{Cascade double Auger process. Identification of the $\mathrm{Ar}^{2+}$ intermediate states}

An enlarged and higher resolution view of the low energy double Auger electrons in Fig. 6 is reported in Fig. 7 (top). Intense peaks due to cascade Auger decays dominate the spectrum and are present with similar branching ratios in the $2 \mathrm{p}_{3 / 2}$ and $2 \mathrm{p}_{1 / 2}$ decay spectrum. This demonstrates that these peaks correspond to electrons emitted in the second step of the Auger cascade. Identification of the intermediate $\mathrm{Ar}^{2+}$ states of the Auger cascade is then straightforward, contrary to the case of the Xe $4 \mathrm{~d}$ cascade Auger decay where first and second step Auger electrons had similar energies ${ }^{21}$. A more precise decomposition of the two dimensional double Auger map of Fig. 4, can be done by selecting more precisely the $\mathrm{Ar}^{3+} 3 \mathrm{p}^{-3}$ ${ }^{4} \mathrm{~S},{ }^{2} \mathrm{D}$ or ${ }^{2} \mathrm{P}$ final states, see Fig.7 (bottom). The intermediate $\mathrm{Ar}^{2+}$ states can then be positioned with respect to these levels. The result is reported in Table 1. The strongest cascade path corresponds to an $\mathrm{Ar}^{2+}$ state of $86.31 \mathrm{eV}$ binding energy, autoionizing to the $\mathrm{Ar}^{3+} 3 \mathrm{p}^{-3}{ }^{4} \mathrm{~S}$ ground state with the release of a $2.19 \mathrm{eV}$ electron. The observed $30 \mathrm{meV}$ width for this electron which is of the order of the instrumental resolution suggests a lifetime of the $\mathrm{Ar}^{2+}$ intermediate state longer ( $>22 \mathrm{fs}$ ) than that of the initial $\mathrm{Ar}^{+} 2 \mathrm{p}$ hole (5.5fs corresponding to a $118 \mathrm{meV}$ lifetime broadening ${ }^{35}$ ). That means a fast emission of the first Auger electron of the cascade, followed by a slower emission of the second Auger electron, similar to what was observed for the Xe $4 \mathrm{~d}$ case $^{21}$. Note in Fig. 7 that only a structureless continuum is obtained for the $\mathrm{Ar}^{3+} 3 \mathrm{~s}^{-1} 3 \mathrm{p}^{-2}$ final states, suggesting a mainly direct double Auger path for their formation. 
The contribution of the double Auger process in the complete Auger spectrum of Fig.3 can now be done by reporting projections of the double Auger spectra on $\mathrm{x}$ and $\mathrm{y}$ scales such as the one from Fig. 6 and by scaling these projections to the complete coincidence Auger spectrum with the known detection efficiency. This is done in Fig 8 for the $2 p_{3 / 2}$ Auger decay. The gray contribution comes from double Auger processes populating the $3 \mathrm{p}^{-3}{ }^{4} \mathrm{~S},{ }^{2} \mathrm{D}$ and ${ }^{2} \mathrm{P} \mathrm{Ar}^{3+}$ states only. One observes a perfect agreement with the intensity of the complete Auger spectrum in the energy gap where the $3 \mathrm{p}^{-3} \mathrm{Ar}^{3+}$ states only can be formed, for Auger energies between 151 and $163 \mathrm{eV}$; double Auger decay to more excited $\mathrm{Ar}^{3+}$ states contributes for lower Auger energies. This implies a sharp frontier between the single Auger and the double Auger spectra. In other words, as soon as the double Auger threshold is reached, electrons are emitted in pairs, and $2 \mathrm{p}_{3 / 2}$ Auger electrons of less than $163 \mathrm{eV}$ are no longer associated with the single Auger process. Fig 8 also reveals that the weak Auger line at $165 \mathrm{eV}$ corresponds to the formation of an $\mathrm{Ar}^{2+}$ state with a binding energy inferior to the triple ionization threshold, while peaks at 147.5, 153 and $162 \mathrm{eV}$ are due to $\mathrm{Ar}^{2+}$ states imbedded in the triple ionization continuum. They are resolved thanks to the second step Auger electrons released during their autoionisation, as reported in table 1 and in Fig 8. Comparison with the calculations of Pulkkinen et $\mathrm{al}^{21}$ shows that these $\mathrm{Ar}^{2+}$ states are $3 \mathrm{~s}^{-2}$ correlation satellites states with $3 \mathrm{~s}^{2} 3 \mathrm{p}^{2} 3 \mathrm{~d}^{2}$ configuration. We observe here that, similar to the Xenon $4 \mathrm{~d}$ case $^{21}$, cascade Auger decays comes from intermediate states populated by a 2 electron process involving two 3s electrons. Three electron processes linked with the double Auger decay are here more easily separated than in the Xe $4 \mathrm{~d}$ case, due to a clear energy separation of the energies of the associated Auger electrons.

The probability for a $2 \mathrm{p}$ hole to experience a double Auger decay can be estimated by comparing the coincidence counts for detecting one or two Auger electrons in coincidence with 
the $2 p$ photoelectron. Taking into account the estimated $49 \pm 5 \%$ detection efficiency one obtains a probability for double Auger decay of $9.1 \pm 1 \%$ for $2 \mathrm{p}_{1 / 2}$ and $9.4 \pm 1 \%$ for $2 \mathrm{p}_{3 / 2}$ hole, in reasonable agreement with the $10 \%$ value obtained from electron / ion coincidences by Saito and Suzuki ${ }^{27}$. Note that our value is only indicative because we supposed here the detection efficiency to be independent of the electron kinetic energy. Furthermore it is probably underestimated, as we are blind to Auger electrons with energies in the $(80,95 \mathrm{eV})$ range (see above), which can contribute to the double Auger decay.

\subsection{Triple Auger decay}

Finally, it was possible to detect the weak signal of triple Auger decay from both the $2 \mathrm{p}_{3 / 2}$ and the $2 \mathrm{p}_{1 / 2}$ holes. Fig 9 shows the spectrum of the energy sum of the three Auger electrons, detected in coincidence with either a $2 \mathrm{p}_{1 / 2}$ or a $2 \mathrm{p}_{3 / 2}$ photoelectron. On a binding energy scale, peaks at a kinetic energy of $105 \mathrm{eV}\left(2 \mathrm{p}_{3 / 2}\right.$ decay) and $107 \mathrm{eV}$ ( $2 \mathrm{p}_{1 / 2}$ decay) agree reasonably well with the $143.81 \mathrm{eV} \mathrm{Ar}^{4+}$ threshold from the literature ${ }^{34}$. To the best of our knowledge, it is the first time that is reported a coincidence experiment where four electrons resolved in energy are detected in coincidence. Estimates done with the overall detection efficiencies from Fig.1, from the observed numbers of (photoelectron / three Auger electrons) coincidences imply that $2 p$ holes decay by triple Auger emission with a $0.18 \pm 0.1 \%$ probability. This value is only indicative of the order of magnitude of this process due to the uncertainties in the detection efficiency, and because Auger electrons with energies in the $(80,95 \mathrm{eV})$ range escaped detection here. 


\section{Conclusions}

We have demonstrated the power of multi-coincidence spectroscopy to disentangle the Auger decay paths following $2 \mathrm{p}$ inner-shell ionization of Argon. Even in a case which is not favorable in terms of energy resolution since a fast Auger electron is emitted with a limited resolution of about $3 \mathrm{eV}$ it is possible to reconstruct precisely the double Auger decay paths since a low energy electron is emitted in coincidence with a high energy one. The cascade double Auger decay is well understood in this way. Triple Auger decay, although a minor process, has also been observed for the first time with this technique.

\section{Acknowledgements}

The support of the BESSY staff and in particular of Willy Mahler and Brigitt Zada is gratefully acknowledged. The project was approved by the Bessy committee program (reference ID .05.1.099) and was supported in part by the European Community - Research Infrastructure Action under the FP6 ”Structuring European research Area” Programme (contract R II 3-CT-2004-506008).

\section{Figure caption}

Fig. 1: Resolution of the magnetic bottle time of flight spectrometer as a function of electron energy. It was estimated from He photoelectron spectra. The line $\Delta \mathrm{E} / \mathrm{E}=1.6 \%$ is a fit through the data points.

Fig. 2: Auger spectrum measured at $337 \mathrm{eV}$ photon energy. Accumulation time was 3 hours. The non coincidence spectrum (thick blue line with intensity reported on the right-hand scale) 
contains the contribution of the $2 p_{3 / 2}$ and $2 p_{1 / 2}$ Auger decays as well as the decays of $2 p$ satellite states. These contributions are extracted by considering the Auger electrons detected in coincidence with the corresponding photoelectrons. Coincidence counts are reported on the lefthand scale. Similarity of the count rates with and without coincidence suggests overall detection efficiencies close to $50 \%$. Comparison is given with the non-coincident high resolution $\mathrm{L}_{2,3} \mathrm{MM}$ Auger spectrum from Pulkkinen et $\mathrm{al}^{24}$, obtained with $265 \mathrm{eV}$ photons (bottom panel). The insert shows the present non coincident photoelectron spectrum, from which we deduce a 1:2.2 ratio for the $2 p_{1 / 2}$ and $2 p_{3 / 2}$ peaks. The same ratio is obtained within error bars when we consider a coincident photoelectron spectrum (not shown), it is close to the statistical ratio of 1:2.

Fig. 3: $2 \mathrm{p}_{3 / 2}$ (red line) and $2 \mathrm{p}_{1 / 2}$ (black line) Auger spectra obtained in coincidence with the respective photoelectron. They are plotted as a function of the binding energy of the final $\mathrm{Ar}^{2+}$ states, by using the reported value for the $2 p$ energies from King et $\mathrm{al}^{29}$. The 2.2 ratio of the $2 \mathrm{p}$ photoelectron components has been used to scale the y axis, see Fig. 2 .

Fig. 4: (color on line) Decay of Argon 2p holes by emission of two Auger electrons. Auger double continua are filtered by the coincidence detection of the corresponding photoelectron: only events where all three electrons of the process are detected are considered here. The two dimensional Auger maps are discretized with a $0.5 \mathrm{eV}$ step. Maximum count rate is $228\left(2 \mathrm{p}_{1 / 2}\right.$ case) or $477\left(2 p_{3 / 2}\right)$.

Fig. 5: Histograms of the sum of the two Auger energies following a $2 p$ hole, as deduced from integration along the diagonal lines of Fig. 4. The graphs are plotted as a function of the binding energy of the final $\mathrm{Ar}^{3+}$ states, by using the value for the $2 \mathrm{p}$ energies from King et $\mathrm{al}^{29}$. Vertical bars correspond to reported values of $\mathrm{Ar}^{3+}$ levels ${ }^{34}$. The 2.2 ratio of the $2 \mathrm{p}$ photoelectron components has been used to scale the y axis, see Fig. 2 . 
Fig. 6: One-dimensional double Auger spectra associated with the formation of the $\mathrm{Ar}^{3+} 3 \mathrm{p}^{-3}$ states. They are obtained by projecting the corresponding diagonal lines of Fig. 4 on both the $x$ (fast Auger electron) and the y axis (low energy Auger electron). The 2.2 ratio of the $2 p$ photoelectron components has been used to scale the y axis, see Fig. 2.

Fig. 7: (top) Enlargement of the low energy part of the one-dimensional double Auger spectra from Fig. 6. (bottom). Filtering of the $2 \mathrm{p}_{3 / 2}$ one-dimensional double Auger spectrum is done by considering the $\mathrm{Ar}^{3+} 3 \mathrm{p}^{-3}\left({ }^{4} \mathrm{~S}\right)$ or $\left({ }^{2} \mathrm{P}\right)$ final states. The 2.2 ratio of the $2 \mathrm{p}$ photoelectron components has been used to scale the y axis, see Fig. 2 . The one-dimensional $2 \mathrm{p}_{3 / 2}$ double Auger spectrum associated with the $\mathrm{Ar}^{3+} 3 \mathrm{~s}^{-1} 3 \mathrm{p}^{-2}$ final states is also included. Discretisation by $10 \mathrm{meV}$ step was adopted for all histograms. Note that false coincidences contribute strongly for electron energies close to zero, due to the pile up of electrons of long time of flight. These false coincidences have been subtracted here (and also for the curves presented in Fig. 6); they were estimated by considering ranges for the sum of 'Auger' electron energies out of the coincidence peaks in Fig.5.

Fig. 8: Complete $\operatorname{Ar} 2 p_{3 / 2}$ Auger spectrum from Fig. 2 or 3, compared with the one dimensional double Auger spectrum of Fig. 6. In order to allow comparison, they have been scaled by taking into account the known efficiency detection (Fig.1). Thick vertical bars correspond to the $\mathrm{Ar}^{2+}$ states involved in the cascade double Auger process, they are resolved thanks to the observation of their secondary decay (see text and table1). The position of $\mathrm{Ar}^{3+}$ levels is from reference 34 . Fig. 9: Triple Auger decay of the $2 p$ hole. Histograms give the sum of the three Auger energies when detected in coincidence with a $2 \mathrm{p}_{1 / 2}$ or $2 \mathrm{p}_{3 / 2}$ photoelectron. The 2.2 ratio of the $2 \mathrm{p}$ photoelectron components has been used to scale the y axis, see Fig. 2. The vertical bar corresponds to reported values of the $\mathrm{Ar}^{4+}$ threshold ${ }^{34}$. 
Table 1: Properties of $\mathrm{Ar}^{2+}$ states implied in $2 \mathrm{p}$ cascade Auger decays. Intensity refers to the percentage of the double Auger decay implying this intermediate step, and is calculated from the $2 \mathrm{p}_{3 / 2}$ double Auger spectrum. Assignment comes from Pulkkinen et $\mathrm{al}^{24}$.

\begin{tabular}{llll|ll} 
Binding energy & Intensity & Decay to & \multicolumn{2}{c}{ First Auger kinetic energy } & Assignment \\
$2 \mathrm{p}_{1 / 2}$ & $2 \mathrm{p}_{3 / 2} \quad 2 \mathrm{p}_{3 / 2}$ & from ref. 21 & \\
85.95 & $0.65 \%$ & ${ }^{4} \mathrm{~S}$ & 162.68 & & \\
86.31 & $3.5 \%$ & ${ }^{4} \mathrm{~S}$ & 162.32 & $162.3(\mathrm{expe})$ & $3 \mathrm{~s}^{2} 3 \mathrm{p}^{2} 3 \mathrm{~d}^{2}$ \\
86.58 & $0.4 \%$ & ${ }^{4} \mathrm{~S}$ & 162.05 & $161.6($ theo) & \\
87.98 & $0.6 \%$ & ${ }^{4} \mathrm{~S}$ & 160.65 & & \\
& & & & & \\
92.76 & $0.3 \%$ & ${ }^{2} \mathrm{P}$ & 155.87 & & \\
95.33 & $1 \%$ & ${ }^{2} \mathrm{P}$ & 153.3 & & \\
96.94 & $1.7 \%$ & ${ }^{2} \mathrm{P}$ & 151.69 & 149.99 (theo) $3 \mathrm{~s}^{2} 3 \mathrm{p}^{2} 3 \mathrm{~d}^{2}$
\end{tabular}

\section{References}

1 P. Lablanquie, J. H. D. Eland, I. Nenner, P. Morin, J. Delwiche and M. J. Hubin-Franskin, Phys. Rev. Lett. 58, 992 (1987).

2 P. Bolognesi, M. Coreno, G. Alberti, R. Richter, R. Sankari and L. Avaldi, J. Electron Spectroscopy Rel. Phenomena 141, 105 (2004).

${ }^{3}$ A. Huetz and J. Mazeau Phys. Rev. Lett. 85, 530 (2000)

4 J Ullrich, R Moshammer, A Dorn, R Dorner, L Ph H Schmidt and H Schmidt-Bocking Rep. Prog. Phys. 66, 1463 (2003).

5 G. King and L. Avaldi J. Phys. B. 33 , R215 (2000)

${ }^{6}$ F. Penent, R.I. Hall, R.Panajotovic, J.H.D. Eland, G. Chaplier and P. Lablanquie Phys. Rev. Lett. 81, 3619 (1998) 
7 Th.Weber, A. Czasch, O. Jagutzki, A. Muller, V. Mergel, A. Kheifets, J. Feagin, E. Rotenberg, G. Meigs, M. H. Prior, S. Daveau, A. L. Landers, C. L. Cocke, T. Osipov, H. SchmidtBocking, and R. Dorner, Phys. Rev. Lett. 92, 163001 (2004).

8 M. Gisselbrecht, M. Lavollee, A. Huetz, P. Bolognesi, L. Avaldi, D. P. Seccombe, and T. J. Reddish, Phys. Rev. Lett. 96, 153002 (2006).

9 B. Kämmerling and V. Schmidt, Phys. Rev. Lett. 66, 1848 (1991).

${ }^{10}$ G. Stefani, R. Gotter, A. Ruocco, F. Offi, F. Da Pieve, S. Iacobucci, A. Morgante, A. Verdini, A. Liscio, H. Yao, R.A. Bartynski J. Electron Spectroscopy Rel. Phenomena 141, 149 (2004).

11 J. Viefhaus et al., Phys. Rev. Lett. 80, 1618 (1998).

12 P. Lablanquie, F. Penent, R.I. Hall, H. Kjeldsen, J.H.D. Eland, A. Muehleisen, P. Pelicon, Z. Smit, M. Zitnik and F. Koike Phys. Rev. Lett, 84, 47 (2000)

13 S. Rioual, B. Rouvellou, A. Huetz and L. Avaldi Phys. Rev. Lett. 91, 173001 (2003).

14 N Scherer, H Lorch, T Kerkau and V Schmidt, J. Phys. B 37, L121 (2004).

15 S Sheinerman, P Lablanquie, F Penent, J Palaudoux, J H D Eland, T Aoto, Y Hikosaka and K Ito J. Phys. B. 39 , 1017 (2006)

16 P. Lablanquie, S. Sheinerman, F. Penent, R.I. Hall, M. Ahmad, Y. Hikosaka and K. Ito, Phys. Rev. Lett, 87, 053001(2001)

17 J. Vief haus, S Cvejanovic, B Langer, T Lischke, G Prumper, D Rolles, A V. Golovin, A N. Grum-Grzhimailo, N M. Kabachnik, and U Becker Phys. Rev. Lett. 92, 083001 (2004).

18 J. H. D. Eland, O. Vieuxmaire, T. Kinugawa, P. Lablanquie, R. I. Hall and F. Penent Phys. Rev. Lett, 90, 053003 (2003)

19 P. Kruit and F. H. Read, J. Phys. E 16, 313 (1983).

20 J.H.D Eland, J. Electron Spectroscopy Rel. Phenomena 144, 1145 (2005). and references included

21 F. Penent, J. Palaudoux, P. Lablanquie, L. Andric, R. Feifel and J.H.D Eland Phys. Rev. Lett, 95, 083002 (2005)

${ }^{22}$ Y. Hikosaka, T. Aoto, P. Lablanquie, F. Penent, E. Shigemasa and K. Ito, Phys. Rev. Lett, 97, 053003 (2006)

23 W. Mehlhorn and D. Stalherm Z. Phys. 217, 294 (1968)

24 H Pulkkinen, S Aksela, O-P Sairanen, A Hiltunen and H Aksela, J. Phys. B 29, 3033 (1996).

25 A G Kochur, V L Sukhorukov, A I Dudenko and Ph V Demekhin J. Phys. B 28, 387 (1995).

26 T. A. Carlson and M. O. Krause Phys. Rev. Lett, 17, 1079 (1966)

27 N. Saito and I. H. Suzuki J. of Phys. Soc. Japan 66, 1979 (1997).

28 F. Penent, P. Lablanquie, R.I. Hall, J. Palaudoux, K. Ito, Y. Hikosaka, T. Aoto and J.H.D. Eland, J. Electron Spectroscopy Rel. Phenomena 144, 7 (2005).

${ }^{29}$ G.C. King, M. Tronc, F H. Read and RC. Bradford, J. Phys. B 10, 2479 (1977).

30 B. Eriksson, S. Svensson, N. Martensson and U. Gelius J. Phys. B. 21 , 1371 (1988)

31 S. Svensson, B. Eriksson, N. Martensson, G. Wendin and U. Gelius J. Electron Spectroscopy Rel. Phenomena 47, 327 (1988)

32 P. Glans, R. E. LaVilla, M. Ohno, S. Svensson, G. Bray, N. Wassadahl and J. Nordgren Phys. Rev. A 47, 1539 (1993).

33 S. Ricz, A. Kover, M. Jurvansuu, D. Varga, J. Molnar, and S. Aksela Phys. Rev. A 65, 042707 (2002). 
34 National Institute of Standards and Technology (NIST) Atomic Spectra Database Energy Levels Data available online at http://physics.nist.gov/cgi-bin/AtData/levels_form (2006)

35 M. Jurvansuu, A. Kivimaki, and S. Aksela, Phys. Rev. A 64, 012502 (2001).

\section{FIGURES}




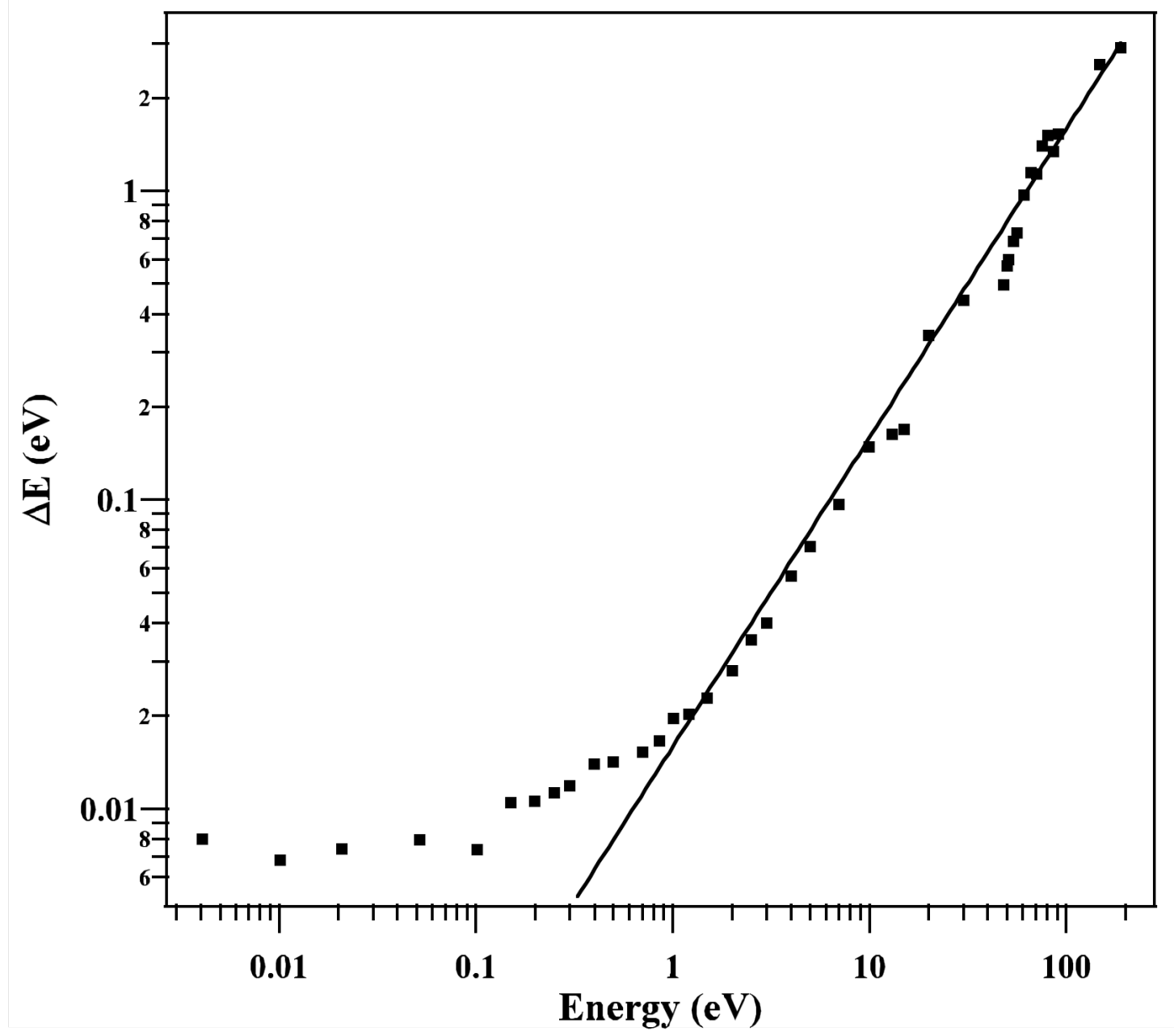

Figure 1 


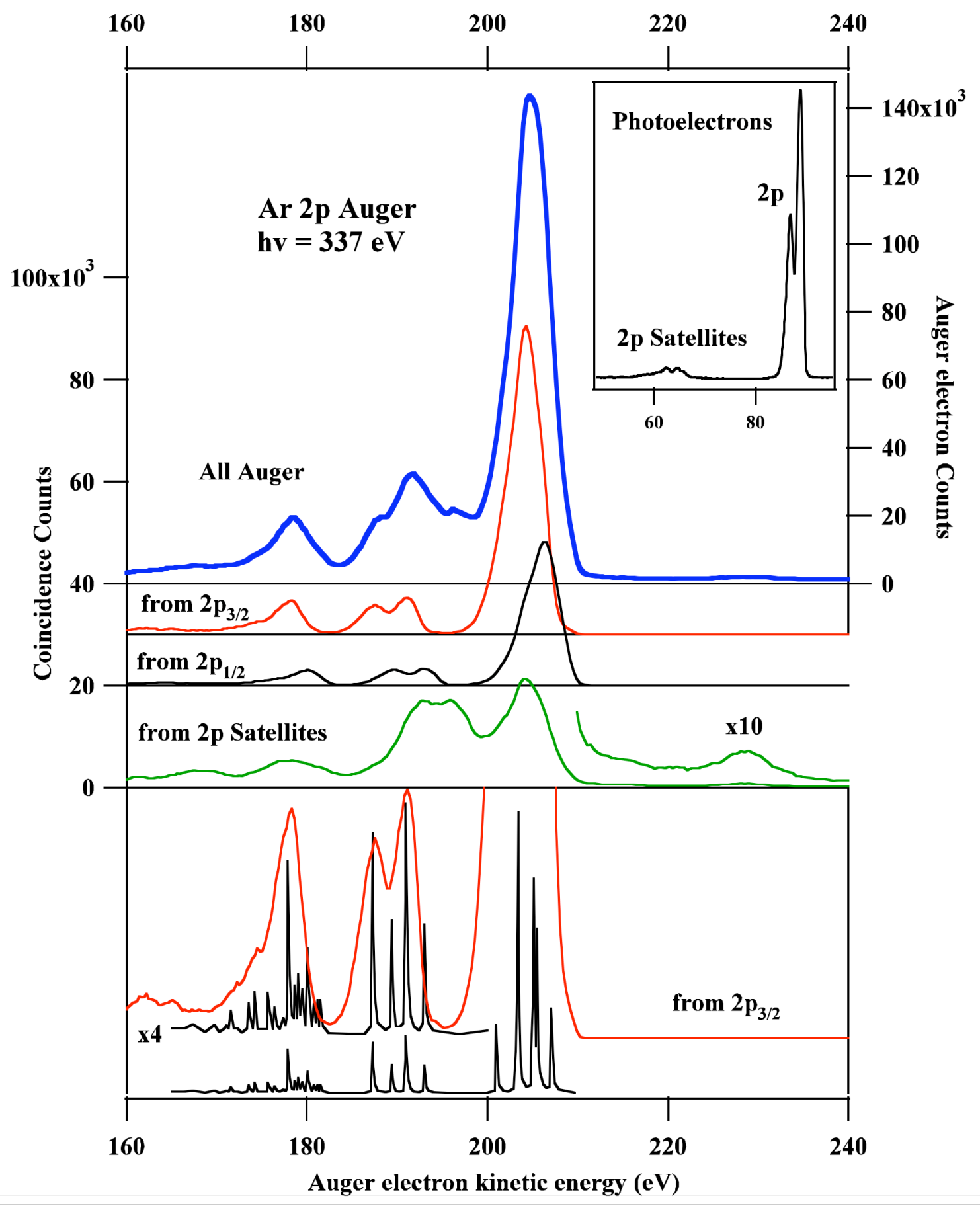

Figure 2 


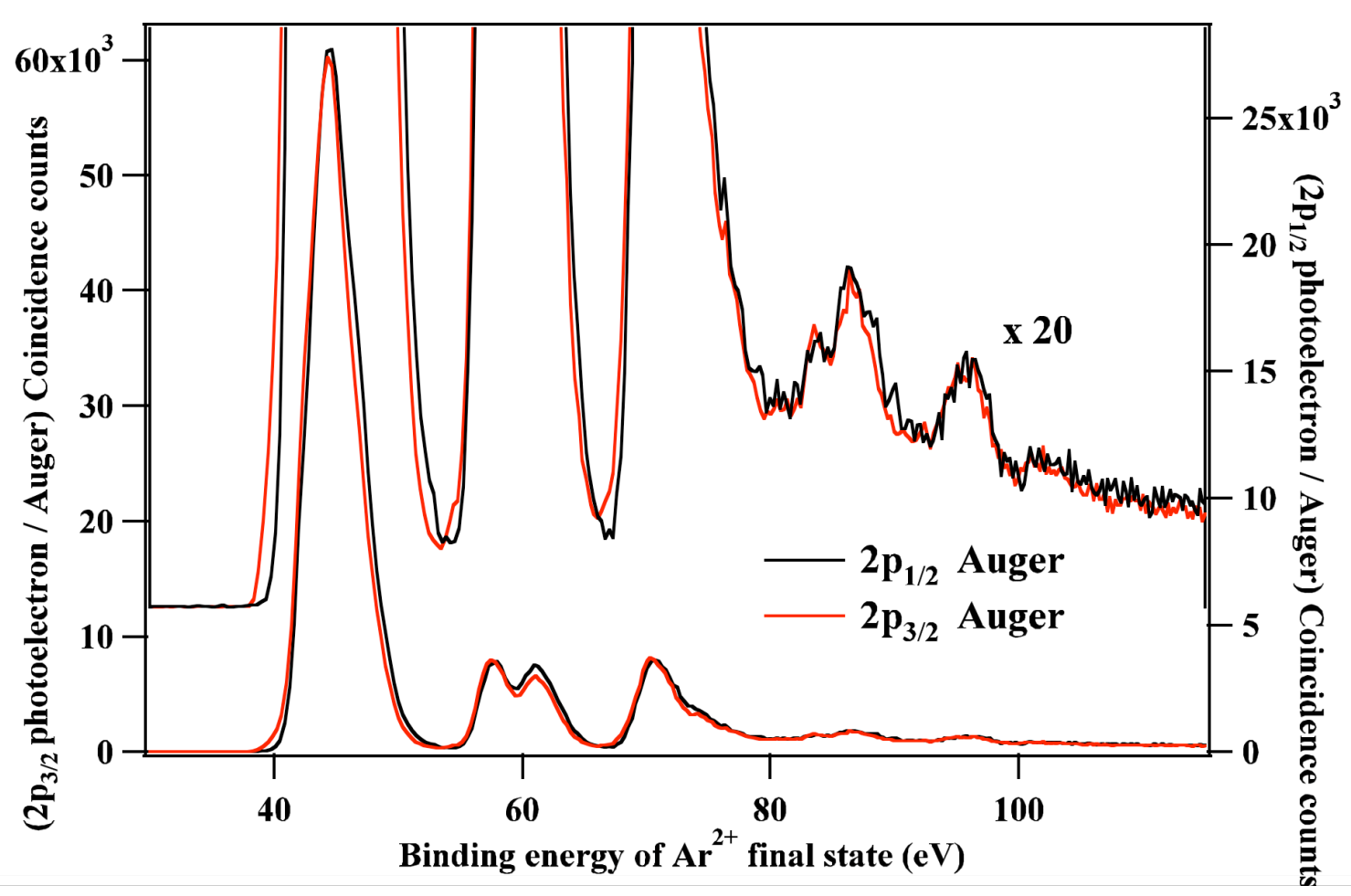

Figure 3 


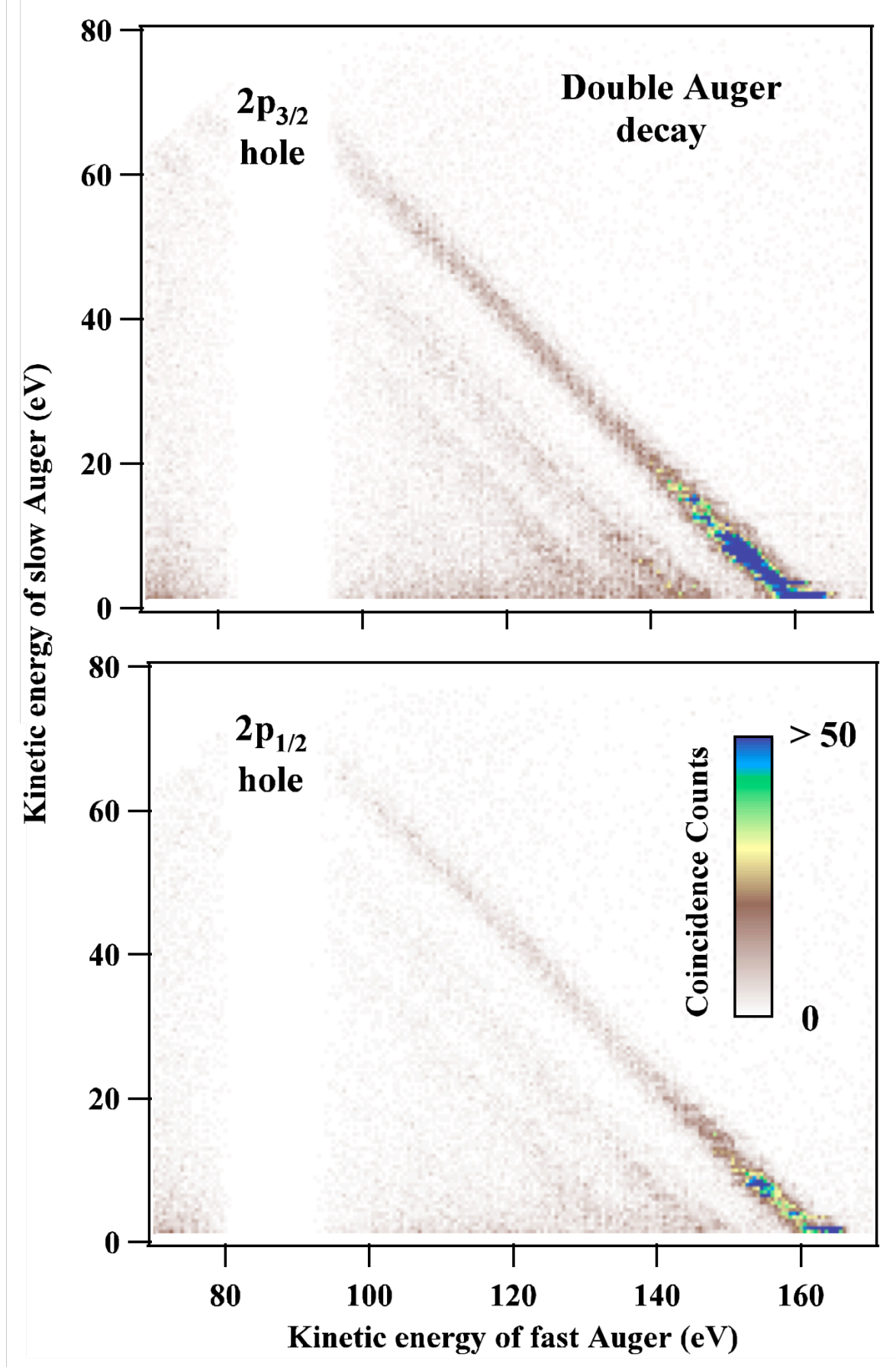

Figure 4 


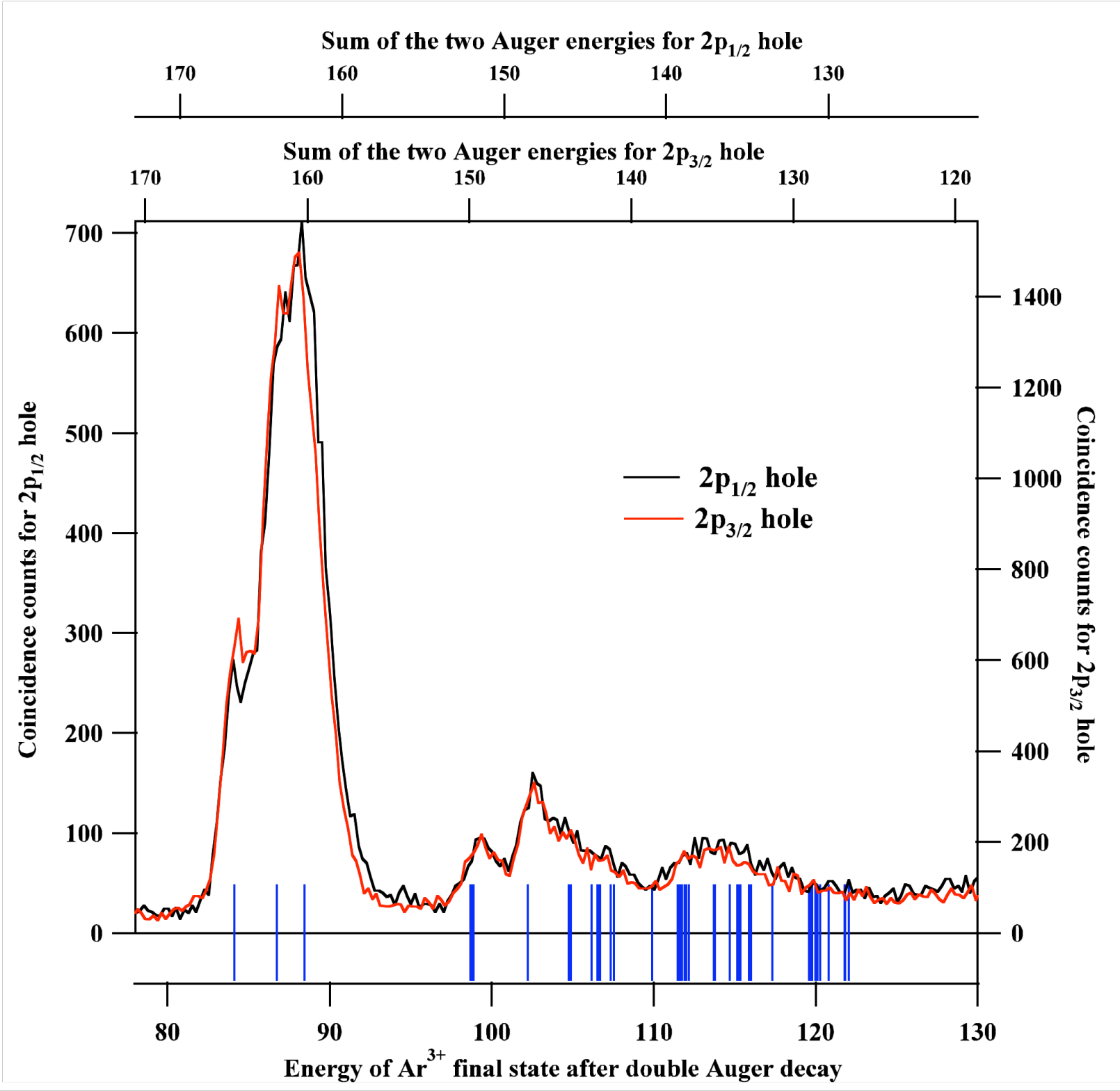

Figure 5 


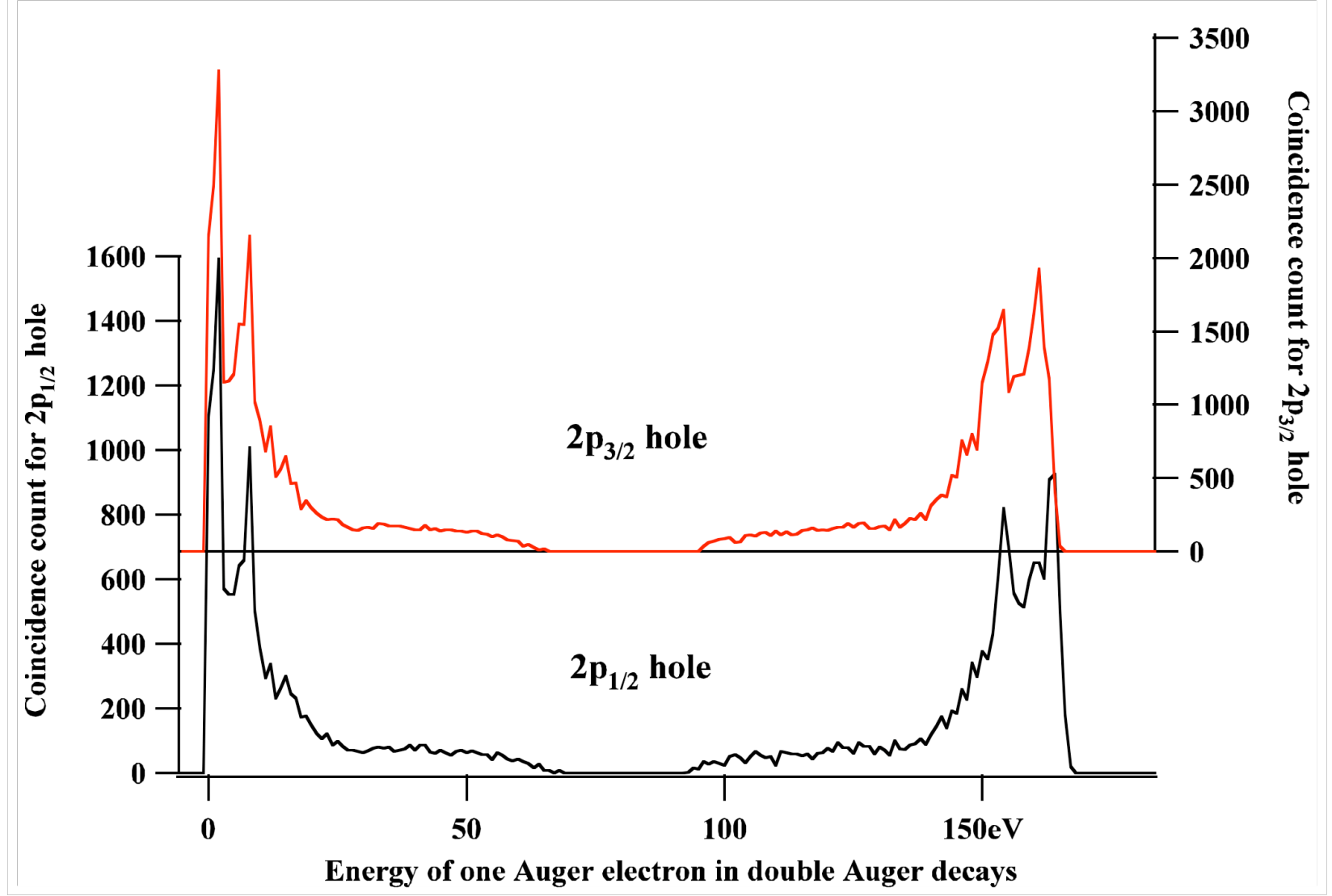

Figure 6 


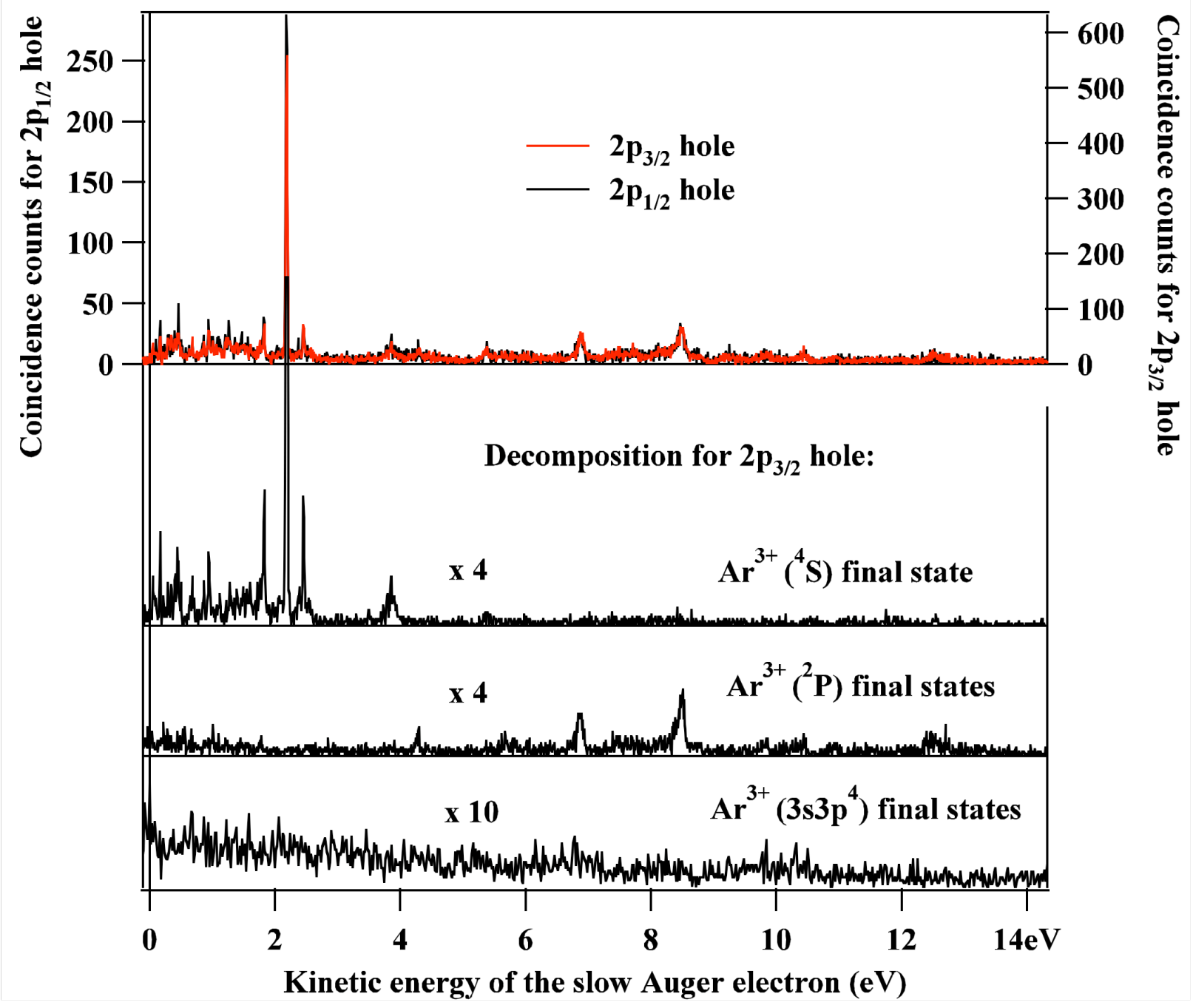

Figure 7 


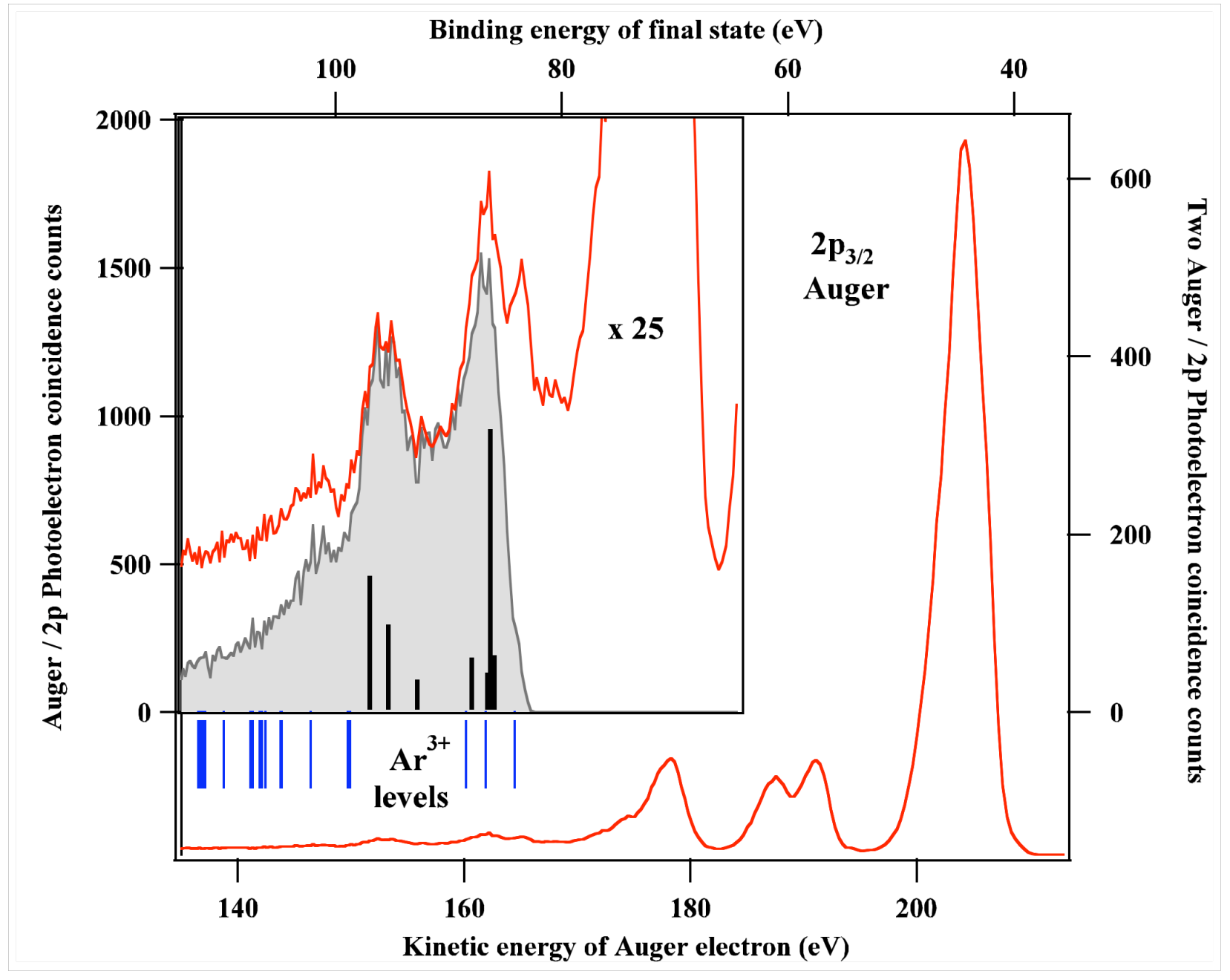

Figure 8 


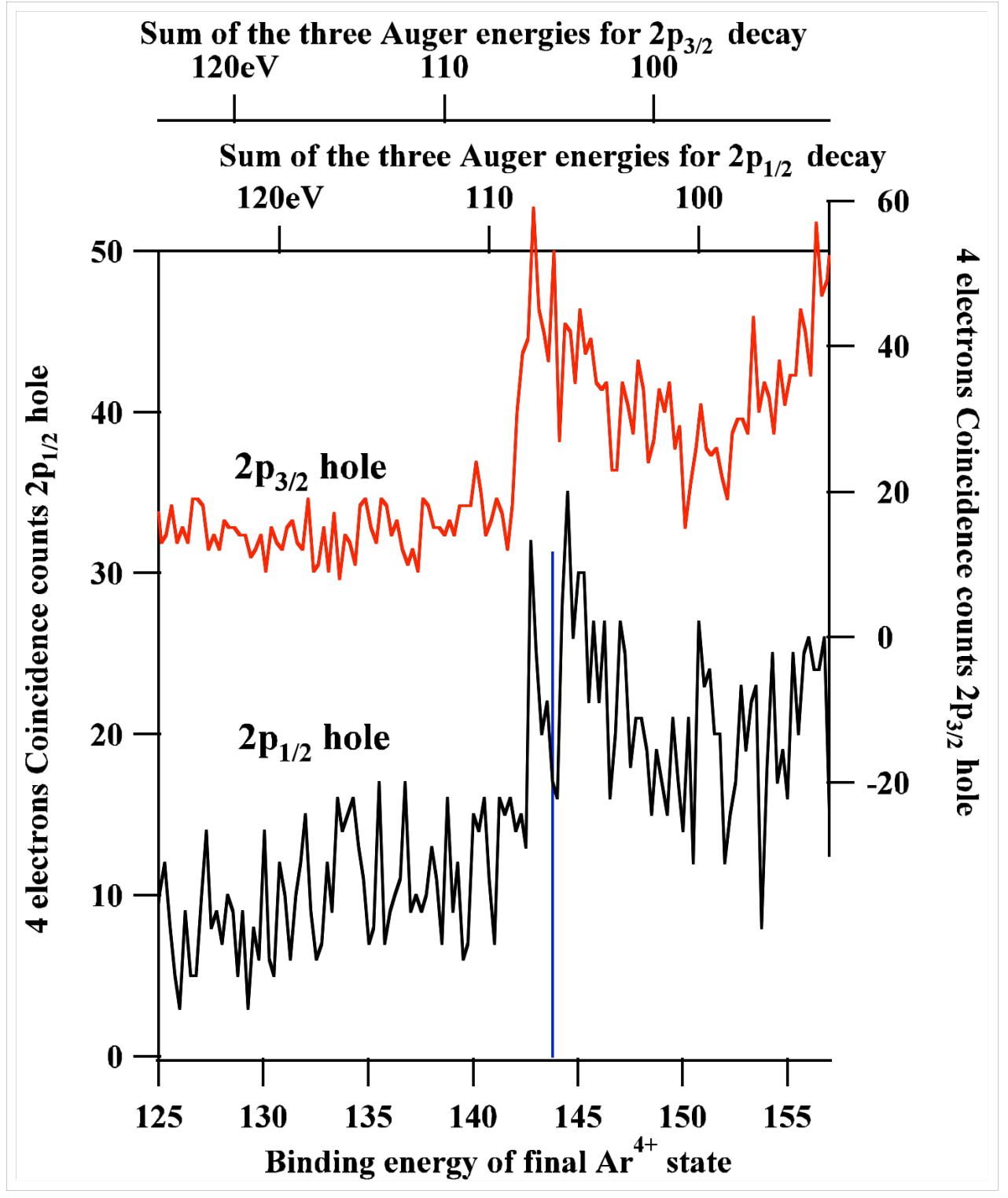

Figure 9 